\title{
The Impact of Community-Based Dental Education on Students
}

\author{
Ronald P. Strauss, D.M.D., Ph.D.; Margot B. Stein, Ph.D.; Jeffrey Edwards, M.S.W.; \\ Kevin C. Nies, M.Ed.
}

Abstract: Community-based dental education (CBDE) shifts a substantial portion of dental clinical education from dental school clinics to mainly public health settings. For dental students to learn effectively in community settings they need preparatory education in cultural awareness, communication skills, and the social and behavioral sciences. The effective integration of CBDE into a dental curriculum requires reflective components, evaluation, and highly organized community-based experiences. This chapter reviews organizational principles and specific strategies to ensure that CBDE is conducted in a fashion that enables student learning and community oral health service. CBDE has substantial potential for affecting the values and behaviors of dental students relative to health care access for underserved populations and for attracting diverse students to dental education. CBDE also provides dentistry with an opportunity to guide dental faculty and student values and orientation towards public service, engagement, ethics, and the health of the public.

\begin{abstract}
Dr. Strauss, Executive Associate Provost at the University of North Carolina at Chapel Hill where he holds joint appointments in dentistry and medicine, served as the principal investigator for the Dental Pipeline Project at UNC; Dr. Stein holds joint appointments at the School of Dentistry and School of Medicine, University of North Carolina at Chapel Hill; Mr. Edwards is a second-year doctoral student in the School of Social Work, University of North Carolina at Chapel Hill; and Mr. Nies is Associate Director of Admissions, University of Maryland School of Nursing. Direct correspondence and requests for reprints to Dr. Ronald P. Strauss, University of North Carolina at Chapel Hill, 104 South Building, Campus Box 3000, Chapel Hill, NC 27599-3000; 919-962-4510 phone; ron_strauss@unc.edu.
\end{abstract}

Keywords: community-based dental education, dental students, access to dental care

$\mathrm{T}$ This chapter examines how schools of dentistry can structure student preparation, student support, and the curriculum to maximize the impact of community-based dental education (CBDE) on students, their values, and their career plans.

\section{Foundations for Student Community-Based Learning}

The ongoing crisis in access to oral health care in America, combined with a growing commitment to greater diversity among dental students, has led to a renewed examination of how dental education prepares students to work with diverse patient populations. Questions have been raised about the locus, timing, and content of instruction that readies students for providing care in underserved communities. Research suggests that when compared with dental students trained solely in traditional settings, students trained in community-based settings demonstrate not only the same achievement of competencies but "appreciably higher clinical productivity and on-time graduation rates."1 Based to some degree on lessons learned from community-based medical clerkships, ${ }^{2-4}$ a growing number of U.S. dental schools have turned to dental rotations or "externships" in publicly funded, community-based clinics as an appropriate setting for clinical training. These CBDE experiences expose dental students at a formative time in their education to the challenges and rewards of treating diverse, underserved populations.

Introducing students to such opportunities in the early years of their education appears to shape how they define their careers and their ethical responsibilities. As students have moved beyond dental school walls, it has become apparent that, to perform optimally in community settings, they require additional training in areas beyond their indispensable technical skills.

CBDE requires enhanced education in communication skills, professionalism, and cultural awareness. ${ }^{5-7}$ The social, behavioral, community, and public health sciences provide key intellectual and interdis- 
ciplinary contributions to this educational process, facilitating students' understanding and enriching their experience in delivering clinical care in communities. ${ }^{8}$ These sciences provide the intellectual foundation for dental students working in the community and for their ability to evaluate and reflect critically on their community-based experiences.

Experiences from the University of North Carolina at Chapel Hill School of Dentistry (UNC) program help demonstrate the educational components. UNC students can go to over seventy publicly owned, underserved sites in diverse communities. These include prisons, nursing homes, psychiatric hospitals, veterans' hospitals, institutions for persons with severely impaired cognitive functioning, and community health centers. The sites provide a venue for clinical dental experiences in settings where students can observe and learn about different patient groups and varied practice patterns. The North Carolina State Dental Practice Act does not permit students to deliver care in private dental offices, which UNC views as a strength. Placement in publicly owned facilities ensures that students are experiencing delivery systems that will not use their labor for personal profit and allows students to appreciate addressing a community need in an underserved setting. All dental students at UNC spend between eight and twelve weeks in extramural rotations (generally four weeks per rotation), with some taking elective summer externships for several additional months. Aside from the local and regional extramural programs, the school sponsors international activities in health services research and clinical care delivery.

\section{Support of Students Working in the Community}

Schools of dentistry generally have a preclinical social, behavioral, and community sciences curriculum that may be oriented towards preparing students for effectively working in, and with, diverse communities. ${ }^{9}$ In order to maximize student learning and community benefit, it is critical that students enter the community with a mind-set and skills that allow them to be open to different cultures and alternative world views. Informed by the social and behavioral sciences, students can see how a dental professional relates to the social and ethical environment surrounding and involving dental practice - the public, patients, other professionals, and staff. Student knowledge about the organization of care delivery and the social, ethical, or political concerns that affect oral health care across all clinical settings is foundational. ${ }^{10}$

Reflective methods help students consolidate CBDE learning. For example, UNC students are required to keep and submit written and photographic journals and scrapbooks, reflective essays, detailed $\operatorname{logs}$ of their daily activities with patients, and an evaluation. ${ }^{11}$ They are also required to attend final reflective sessions facilitated by faculty members associated with the program. These reflections provide opportunities to reinforce and highlight community-level learning experiences..$^{12}$ Recently, UNC experimented with electives in book readings that are conducted during rotational periods, which permit writing and group discussion upon return from the field. Carefully selected book readings of fiction or nonfiction that explore issues of community relevance are a unique opportunity to enrich student learning.

An innovation introduced at UNC is a studentgenerated pledge that is signed by first-year dental students. The pledge states, "As access to dental health care becomes an increasing concern for dentists and their patients, we, the graduating class of the UNC-Chapel Hill School of Dentistry in the year 2007, pledge to commit as dental professionals a minimum of 4 service hours each month to the underserved population in a method most conducive to that community." Students are also advocating that practitioners in the state sign the pledge and engage in increased community service. ${ }^{13}$

Dental students must have basic threshold clinical technical skills before they work at a community site. However, this is not sufficient for an excellent CBDE experience, and sites must be prepared to meet the instructional needs of students. Different schools take very different strategies about what is sufficient dental clinical preparation before community entry. In some dental schools, students are expected to be well along in their clinical skills and to have demonstrated proficiency by a competency test before they are granted leave from the school's clinics for a community site. Other schools see community sites as locales for the development of clinical skill and competence. Such schools ask students to successfully complete some preclinical didactic materials and courses before community placement, but do not expect clinical proficiency. Either way, students have been given the essentials of office infection control, injury/needle stick management, ethics, and clinical history-taking. 
It is important to provide preceptors for externship site fields with a clear idea of the ability level of students who are being placed in their midst. This information allows the preceptor to make clinical case selections and to know what is reasonable to expect in terms of need for supervision. In fourth-year rotations, it is desirable to encourage preceptors to somewhat restrain their oversight to allow advanced students a chance to self-assess their actual capacities and limitations. We find that the finest preceptors ask students a simple question: "What is it you need to learn to be prepared for community practice or graduation?" Students can often give the preceptor an accurate picture of their clinical strengths and weaknesses, thus facilitating growth and learning.

As in all dental training contexts, rotational sites must preserve high-quality care delivery. Preceptors should be asked to notify the school if a student has demonstrated poor judgment or limited clinical ability. On rare occasions, the school may wish to recall a student to place him or her back into the school's in-house clinics for remedial instruction. However, in general, student limitations can be dealt with effectively in community settings because there is often a ready supply of patients in need and an efficient delivery system in place. Preceptors are also encouraged to call the school if an injury, complication, or needle stick has occurred with a student clinician. The hallmark of a fine rotational program is the ability to maximize communication of the school, site, and student when stress or need is evident. Students also should be asked to call the school if their experience in the field site is not to their benefit. This allows the school to maintain quality control and to ensure that students are safe and working in a truly patient-centered care delivery system.

\section{Cultural Awareness and Preparation}

Prior to providing dental services to populations in need, CBDE programs call for specific academic and curricular preparation that is in addition to the traditional emphasis on technical competence..$^{14,15}$ A growing number of dental patients are working poor, unemployed, elderly, disabled, or immigrants for whom culture and income can be a barrier to obtaining oral health care. In order to meet the central goals of community-based dental clinics - to increase the number of dental patients seen and the services provided and to offer patient-centered care - dental students need to be able to interact and communicate effectively and comfortably with their patients. This requires a comprehensive understanding of their patients' lives, including economic, educational, social, and cultural background.

The growing number of immigrant dental patients across the United States in rural and urban areas has lent particular urgency to educating dental students about culture. The marked increase in the Hispanic community in the United States includes many who speak Spanish at home and have difficulty understanding English. Barriers to dental services may be both linguistic and cultural. In addition to becoming more familiar with Hispanic cultures, dental students can surmount a language barrier by learning Spanish, obtaining the services of an interpreter, or translating key documents such as informed consent and written treatment instructions. Cultural awareness furthers patient rapport and cooperation, thereby increasing patient adherence with treatment. Cultural awareness promotes an ethical perspective by honoring patient autonomy, including the right to one's own cultural orientation in interchanges regarding dental care. Teaching cultural awareness is likely to benefit all dental students and patients. ${ }^{16}$

The goal of producing dental school graduates who are technically competent and who possess good communication skills and cultural sensitivity poses challenges for the traditional dental school curriculum with its focus on technical competence grounded in the basic and clinical sciences. Historically, dental education has tended to rely on an approach to service delivery that viewed the dentist as the dispenser of care and the patient as a passive recipient. A behavioral management approach was a way to help the dental practitioner cope with patient behaviors that hindered the rapid and efficient completion of treatment. So, theoretically, dental students were taught how to manage or control patient behaviors and make sure patients follow instructions.

Starting in the 1970s, health practitioners began to question whether the traditional approach to service delivery was effectively achieving its goals. Drawing on the social and behavioral sciences, dentists and other health providers began to publish research indicating that a less hierarchical and more dialogue-based collaboration with the patient about the goals, methods, and desired outcome of treatment was a far more effective tool for obtaining positive treatment outcomes and promoting disease prevention. ${ }^{17}$ The researchers posited that this more holistic 
approach was more suited to patients with little experience with dentists and preventive oral care who were living in difficult social and economic circumstances and accustomed to cultural and linguistic barriers to dental care. In addition, the patient-centered model of health care delivery was more consistent with the goals of community-based medical and dental clinics.

In retrospect, it seems inevitable that an increased emphasis on comprehensive patient-centered dental care would add social science courses to the basic science and clinical skills curriculum of the dental school. The central goal of the social science courses is to teach students how to combine the scientific principles and technical expertise of dentistry with the patient's life history, behaviors, and clinical presentation. ${ }^{15} \mathrm{~A}$ few dental schools began to offer elective seminars in the humanities, borrowing from medical schools where literature was used to expand the frame of reference of their students. ${ }^{18}$ The students in the dental humanities courses were encouraged to view individual patients as people embedded in a dynamic community life with multiple and sometimes conflicting roles and responsibilities. Students and teachers discussed ethics and values and ways to resolve differences between the dentist and the patient.

Another teaching strand emerged that focused on communicating more effectively with patients by increasing awareness among students of the cultural variables that directly and indirectly affect care. Courses focused on social interaction and communication, collaboration, and group problem-solving. ${ }^{15}$ However, these methodologies seemed distant to the framework of traditional dental education and were sometimes greeted by faculty members and students as unscientific, poorly supported by research, conceptually nebulous, and lacking the objectivity associated with basic science.

Currently, the cultural and social sciences play a more pivotal role in preparing dental students and residents for community rotations and the care of diverse community-based populations. Aspects of the curriculum that deal with models of delivery of care, the epidemiology of oral disease, and public health dentistry focus on populations and enable the dental student to place the individual patient in a broader societal perspective.

Courses that focus on developing patient/provider communication skills foster an understanding of the cultural variables that directly and indirectly affect the delivery of community-based and patientcentered care. These skills are based on empathy, curiosity, intellectual flexibility, heightened aware- ness, and willingness to respect, listen, and learn from the patient.

Interpersonal communications skills training has been swiftly integrated into the dentistry curriculum. ${ }^{17}$ The communications field possesses an established methodology drawn from the social sciences, which provides legitimacy and makes it simple for instructors with little formal training in communications to apply it to their courses. In addition, by 2000, there was a growing body of research that evaluated various teaching methodologies in communications and assessed the learning outcomes for students and the benefits in terms of treatment outcomes in patients. ${ }^{17}$

There is a general consensus that active practice in communication skills, with feedback, enhances student ability more effectively than didactic instruction. Student interviews with simulated patients, observations of interaction between more advanced students or faculty members and dental patients, small-group instruction using role-playing, student interviews of real patients, and analysis of videos of dentist/patient interactions are proving to be more effective teaching strategies than lectures or readings. Studies reporting successful communications training interventions with dental students are beginning to appear in the literature. ${ }^{19}$

Dental schools in North America have made considerable progress introducing communication skills training into the curriculum. In a survey of the curricula of forty dental schools in North America (thirty-six in the United States and four in Canada) published in 2002, researchers found that one-third had a separate course focusing mainly on interpersonal communication. ${ }^{17}$ Thirty-eight percent taught one such course; 45 percent had two courses; 10 percent offered three courses; 5 percent had four courses; and one school had six courses that included the topic. The survey also found that many dental schools did not provide gradual exposure to communication starting with basic principles and progressing to more complex concepts. They attempted to teach quite complex issues, such as patient education and managing difficult patients, without a full grounding in communication theory and knowledge of basic skills.

The researchers found that, in general, the methodologies used to teach communication skills in dental schools were problematic in that they strongly favored the less effective passive, didactic/lecture approach over the interactive methodologies. In terms of teaching methodologies, 91 percent used lectures, 27 percent used role-playing, 20 percent used videos 
as a trigger for discussion, and 18 percent used case presentations. Looking at evaluative measures, they found that many of the schools relied primarily on indirect evaluation of communication skills through written and oral tests for evaluating cognitively based skills. Relatively few schools used direct evaluation measures such as simulated practice that more accurately assess actual behavior. The combined use of these complementary evaluation methods was recommended. Nevertheless, there are several outstanding examples of dental schools with wellconceptualized and carefully designed programs for teaching interpersonal communication. At least one dental school offered several courses over years of instruction, used lectures as well as active practice with feedback, and used indirect and direct evaluation methods with written guidelines.

Institutional recognition of the importance of culturally sensitive dental care and its inclusion in the dental curriculum is a relatively recent development, and its implementation remains sporadic across U.S. dental schools. In 2000, the American Dental Education Association (ADEA) formally recognized the importance of culture in the delivery of oral health services and advised dental educators to include "cultural and linguistic concepts" in dental curricula. ${ }^{16,20}$ Dental schools in areas with large immigrant populations, particularly publicly funded institutions or those with a history of investment in public health initiatives, have been trendsetters in this domain. The Robert Wood Johnson Foundation-funded Pipeline, Profession, and Practice: Community-Based Dental Education program, in particular, has articulated programmatic incentives for dental schools to develop culturally competent dental services oriented to diverse and underserved populations. This initiative was founded on the premise that providing culturally aware dental care to underserved populations has both pragmatic and ethical justifications.

Formal inclusion of training designed to increase awareness of different cultures and to teach about culture is a recent phenomenon in U.S. dental schools. It has been pointed out that recognizing that culture is a part of everyone's identity is a key aspect of acquiring cultural awareness. ${ }^{21}$ We have an obligation to appreciate the vulnerability of all patients in health encounters and to recognize bias when it exists. Cultural awareness or competence training is founded on the basic principles of self-awareness, respect for diversity, and sensitivity in communication. Any approach that presents a compendium of behaviors and health beliefs for various ethnic groups is not encouraged as it may unwittingly foster stereotypes.

\section{Communication Skills and CBDE}

U.S. dental schools are witnessing the growth of curricular approaches that teach skills for bridging the cultural gap between patients and practitioners. ${ }^{22,23}$ The approaches teach communication skills using social science methodologies: questionnaires, interviewing, clinically based scenarios, simulated patients, role-playing, and observation. Additionally, methodologies such as journal writing, servicelearning experiences combined with reflection, and photography are tapped to promote self-reflective analysis that is essential to cultural awareness and competence. ${ }^{11,12}$

Several "toolboxes" are available for teaching cross-cultural awareness and communication skills in health care that could be adapted to the particular demands of teaching dental students. ${ }^{24,25}$ They cover topics such as language, health belief systems, values, and assumptions and contain assessment tools for the classroom and clinic. They also describe some of the qualities found in effective diversity trainers: understanding one's own life issues around diversity; knowing one's own stereotypes and biases; being able to tolerate ambiguity; being able to facilitate discussions of highly emotional and potentially conflicting issues; creating a nonthreatening, high-trust environment for learning; and being sensitive to a group's mood or tone. ${ }^{25}$ Many of the suggested exercises, such as role-plays, case studies, critical incidents, and videos, are most effective in small groups. Obviously, given the tightly packed dental curriculum and limited staff resources, instructors will have to be imaginative and flexible in finding ways to use these teaching techniques effectively. In a few cases, where the dental school has made teaching communication skills and/or cultural competence a priority, additional funds and instructors have been provided. In these situations it is feasible to use simulated patients for students to interview, with videotaping of each interaction to permit later analysis and evaluation by the student and an instructor.

Despite the difficult logistics of teaching these skills to dental students in a large classroom, there are teaching methods that can be adapted to large-group settings. Students could be assigned to small working 
groups in which they would role-play, analyze a case in terms of communication and cultural competence criteria, and then present the outcome to the entire class. They could videotape each other, analyze the interactions, and present that to the larger class. Students could also observe dental patient/dental student interactions in the operatory or waiting room, analyzing the interactions according to various criteria. Students also could keep a written journal during their extramural clinical experiences, providing a framework for their observations and reflections.

Evaluating cultural awareness is essential and challenging. It requires time and faculty effort. Many dental schools have used the objective structured clinical examination (OSCE) as an opportunity to assess communication and cultural awareness, either using standardized patients or by having the dental student view a videotaped dentist-dental patient interaction and analyzing it on the basis of established criteria. Asking dental patients to complete a survey about their dental student provider's communication skills and asking the dental student to complete a self-evaluation on the same patient is another strategy. However, evaluating the dental student's cultural awareness using this format remains a challenge.

In summary, preparing dental students for work in community dental clinics requires much more than clinical competence. The importance of teaching communication skills and related skills for achieving cultural awareness and competence has been established in theory. Developing a curriculum that builds on theoretical foundations, systematically teaches both basic and more complex skills, and is articulated throughout all years of dental school is an intellectually fascinating and challenging task that places specific demands on the dental school curriculum and requires an institutional commitment. One of the most important anticipated benefits from dental students' growing participation in communitybased dental clinics will doubtless include better evidence-based instructional methods for helping young dentists become adept in their ability to deliver cross-cultural care.

\section{Reflection and Self- Awareness}

Community experiences provide an outstanding opportunity for education and building self-awareness. However, experience alone without evaluation and reflection may be a missed opportunity. One model for how reflection may be built into community-based dental education occurs at the UNC School of Dentistry. The school requires all fourth-year dental students to spend an average of fifty-nine days in community clinical sites. For all students, the rotation time is divided evenly between a community-based hospital or institutional setting and a community health clinic or program. ${ }^{12}$ UNC has worked to increase the average number of days that senior dental students spend at community sites by offering "capstone experiences" whereby senior students who have already met or are on pace to finish their in-house clinic requirements and academic coursework for graduation may rotate for additional days at approved community sites.

Before dental students leave campus to enter varied community dental settings for clinical rotations, they receive formal preparation regarding the reflective activities they are expected to engage in both during and after completing those rotations. The preclinical curriculum reflects a concerted and global effort to equip all students with a fund of knowledge in basic sciences, community sciences, culturally sensitive communication, and basic clinical skills. The reflective component includes critical incident reports, a critical incident essay, and a photographic scrapbook as well as a post-rotational reflective session. ${ }^{11}$

The course director clearly explains all of the assignments, both reflective and nonreflective, to the students, verbally and in writing. At least one month before the start date of the earliest possible community rotation, the course director holds a mandatory "send-off" meeting with the students. This one-hour meeting is held at the dental school and provides the students with a structure and understanding of key objectives that buoy their confidence. In addition to receiving a verbal review of the assignments and the chance to ask questions throughout the session, the students are given paperwork and instructions related to community rotations. These extensive packets include logbooks, forms for completion, descriptions of all requirements and their due dates, and contact information for UNC personnel. From an HIV needle stick protocol contained on a wallet-sized laminated card to critical incident logs on $8 \frac{1}{2}$ " x 11 " paper, no stone is left unturned. The information is a beacon of sorts so that students at unfamiliar and often remote domestic and international locations never feel marooned, especially in their first few days at the site. 
Students learn that they will be expected to complete weekly critical incident reports during their community rotations. Therefore, the send-off packet includes log sheets with space for students to note critical incidents for each of the weeks. The completion of critical incident reports provides the initial step in the process of encouraging and enabling students to engage in structured reflection during their rotation and lays the groundwork for more in-depth post-rotational reflective activities. Students record the most moving encounters they experienced in a particular rotation week and events during rotations that have challenged them personally, professionally, or both. ${ }^{12}$ The course director instructs students to focus on the issues, feelings, and reactions-positive, negative, or ambivalent-surrounding dental procedures or clinical interactions in the community; the students are not simply to recount the details of dental procedures.

The critical incident reports are to be concise, generally one or two paragraphs in length. The brevity allows students to record the event in the midst of the fast-paced community clinic environment. The requirement to complete logs helps ensure that students become mindful of their experiences. The written documentation of those experiences then becomes available for the students to revisit as they engage in more in-depth reflection while crafting a critical incident essay.

In 2003, researchers drew an explicit link between research on the importance of CBDE for dental students and social science research about the value of reflection in the professional development of health practitioners. ${ }^{12}$ As evidence of the dental profession's increasing commitment to developing community-minded and patient-focused dentists, they cite standards outlined by $\mathrm{ADEA}^{20}$ and the Commission on Dental Accreditation, as well as the increased numbers of dental schools whose curricula include some form of community-based dental rotational experiences. However, the authors contend that professional standards and community experiences alone are not always sufficient to produce dentists who are prepared to provide outreach, empathic patient care, and culturally sensitive communication. Community experiences, whether in dentistry or another field, are likely to be transitory events that do not promote long-term learning when they are not accompanied by reflection..$^{12}$ The reflections of students on their community-based dental experiences are beneficial to their personal and professional growth. The researchers examined dental students' written reflections on their community-based clinical rotations. Then, they applied qualitative analysis to study critical incident essays written by 160 senior dental students at UNC in 1998 and 2000. The results of the analysis indicated that although students wrote independently about their reflections on varied clinical community sites, three common themes emerged: personal and professional growth; enhanced awareness; and commitment to service.

The theme of personal and professional growth alluded to an increased realization of the need to be truly competent and demonstrate respectful and empathetic interactions with patients. Students critically evaluated their own strengths and limitations, including their readiness to deliver patient-oriented care in unfamiliar environments with patients whose backgrounds were often very different from their own. For instance, students wrote of their first dental encounters with incarcerated patients and the feelings associated with first recognizing and then adjusting their preconceived notions about this population. Students also recounted experiences that challenged and broadened their communication skills. It was evident that community rotations offered some students their first opportunity to accomplish very difficult and important communication tasks, such as delivering bad news. Students reported that tackling tough and novel encounters increased their confidence. ${ }^{12}$

In the 2003 article, the authors also highlighted the dental students' increased understanding of the many challenges that confront their patients who were often of low socioeconomic status. These experiences solidified the desires of some students to provide care to the disadvantaged after graduation from dental school. Rather than simply viewing their patients as mouths in need of clinical treatment, the student dentists began to view their patients more holistically as people whose dental needs could not be separated from the broader social webs of poverty and racism that often were the source of those needs. In some instances, students realized that the quality of dental care was compromised for patients with minimal ability to pay. Such realizations were sometimes the catalyst for written reflections upon fundamental equity and professional responsibility. Some reflections addressed the disparity between ideal ethical care and the actual services provided to economically and socially disadvantaged patients. Frequently, pondering such gaps led to student-generated promises to bridge them in their own future practices. ${ }^{12}$

As with the critical incident reports, critical incident essays remain part of the UNC required 
curriculum for community dental rotations. The process of generating critical incident essays is outlined verbally and in writing at the send-off gathering. The completion of critical incident essays is required to pass the community rotations course, though the essays themselves are not graded for content or form. Students are instructed to craft typed essays of two to five pages. Students write the accounts as first-person narratives that draw upon a single event previously recorded in less depth as a specific critical incident report. In order to maintain confidentiality, students use pseudonyms for colleagues and patients. Critical incident reports provide a building block for further reflection in the forum of post-rotational reflection seminars, to be discussed below.

In addition to written narratives, dental students may create photographic scrapbooks, as they do in the Mexico Project (www.unc.dent/mexico), an annual dental service trip during which students provide dental care to the residents of a large Mexican orphanage. ${ }^{11}$ Students use the documentation to elicit further reflection about the meaning of communitybased dental service. The scrapbooks by senior dental students are intended to tell a story about the lessons learned. As with all required community rotational assignments, students are given explicit instructions prior to the start of their rotation and may view samples written by previous students.

After senior students have completed their community-based clinical rotations, critical incident reports, and essays and photographic scrapbooks, one final structured reflective experience remains: the post-rotational reflection seminar. These seminars, like the critical incident essays, revolve around the feelings and issues associated with experiences, rather than the simple recounting of clinical procedures. ${ }^{11}$ In groups of eight to ten, students discuss the critical incidents that occurred during their rotations. Meeting times are selected that do not present curricular or clinic conflicts for students; meeting locations are secured by the course director. Each two-hour group is guided by a trained dental faculty facilitator, selected by the course director, who ensures that each student receives a chance to share insights. The facilitator does not record the responses of individual group members, nor is there an attempt to shape the comments offered by students. This format provides an opportunity for students to explore commonalities and differences they gleaned during their communitybased rotations.

Critical incident reports and essays, photographic scrapbooks, and post-rotational seminars are part of a structured curricular experience that provides dental students with valuable opportunities to reflect upon the lessons learned through engagement in community dental settings. It is important to cultivate the personal and professional growth of dental students through assignments that generate valuable reflection and constant re-evaluation of professional integrity. With an ever-increasing need for dentists who will address dental health disparities, a commitment to service and reflection represents an initial step toward the preparation of communityoriented dentists capable of crossing socioeconomic, racial, and communication boundaries to provide quality care to all.

\section{Impact of CBDE on Schools of Dentistry}

In addition to changing curricular offerings, a commitment to CBDE has the potential to alter the composition of the school's student body, faculty perceptions, and decisions regarding revenue from clinical care. Changes in course offerings, student recruitment strategies, faculty support of community engagement, and clinical practice models vis-à-vis school clinics can influence the very fabric of the institution.

Prior to involvement in the Pipeline program in 2002, UNC evidenced a commitment to CBDE and the recruitment and retention of underrepresented minority (African American, Hispanic, and Native American) students. Nearly four decades before the implementation of the Pipeline program, dental students participated in clinical experiences in the community through Dentistry in Service to Communities (DISC) rotations. The notion of community was expansive and not limited to locations near the school. DISC rotations did and still do include state, national, and international sites with one important commonality: none have been at private dental practices. Likewise, underrepresented minority recruitment programs predated the Pipeline program. Two examples include the Medical Education Development (MED) Program and the Science Enrichment Preparation (SEP) Program. Both MED and SEP programs are academic summer experiences for underrepresented minority students interested in the health professions who have finished their undergraduate studies (MED) or are currently enrolled in an undergraduate program (SEP). ${ }^{26}$ 
The UNC School of Dentistry's participation in the Pipeline program ushered in changes in the school's CBDE rotations, student recruitment strategies, and admissions process. ${ }^{26}$ Involvement in the Pipeline program did not change the character or number of sites formerly available to students through the DISC program, but the time spent by fourth-year students at those rotational venues increased. Prior to the Pipeline program, all senior dental students spent forty days at community sites. By 2007, the average senior dental student spent fifty-nine days. UNC used Pipeline program resources to increase the number of underrepresented minorities interested in dentistry who participated in the MED and SEP programs by funding additional seats in those programs. For underrepresented students accepted to UNC, W.K. Kellogg Foundation funds were used to purchase laptop computers to ease the financial burden of this required resource. ${ }^{26}$ In reference to the dental school's admissions process, a greater reliance on whole-file review was realized. That is, in addition to test scores and grades, respect for and consideration of the social backgrounds and experiences of applicants have been woven into the process. ${ }^{26}$

In addition to increasing the time that dental students participated in community rotations, the national Pipeline program wanted participating schools to increase the number of underrepresented minority students enrolled at their institutions. ${ }^{27}$ This metric for evaluation was chosen in part based on the assumption that by making the composition of dental school student bodies more diverse, a greater number of dental graduates would be more inclined and better equipped to address the access to care issues outside of the schools' walls. Addressing issues of equity and access both inside and outside of higher education is a hallmark of service-learning, of which CBDE is an example..$^{28}$ Data gleaned from evaluations of dental schools participating in the Pipeline program suggest that there is a relationship between increased avenues for service opportunities and the recruitment of underrepresented minority students to dentistry. Even though service appears to be a more important determinant in the attractiveness of dentistry as a profession to underrepresented minority students than to majority students, underrepresented minority students typically have less exposure to the profession. Given that, the Pipeline evaluation team underscored the importance of predental academic enrichment opportunities. ${ }^{29}$

Faculty members play a critical role in the success of CBDE. To sustain service-learning as a long-term part of a school's culture, faculty members must be actively involved in service-learning activities, which must be supported by their institution. One critical area of institutional support is the tenure and promotion evaluation methods of universities. That is, faculty members must be provided with opportunities to learn about incorporating servicelearning into their scholarship and to reap rewards in terms of career advancement for that scholarship as opposed to being penalized by advancement models that do not recognize service-learning initiatives in the promotion and tenure process. Faculty for Engaged Scholarship, which represents collaboration between the Community-Campus Partnerships for Health (CCPH) and the University of North Carolina at Chapel Hill and the University of Minnesota, provides an example of highlighting the role of the faculty in service-learning successes. ${ }^{28}$

Even faculty members and administrators who are not directly involved in CBDE activities are important to sustained success of these initiatives. For instance, faculty and administrative support for extended opportunities for dental students to undertake clinical rotations outside of the schools' in-house clinics is important. ${ }^{30}$ This support is very important for schools seeking to significantly increase the number of opportunities for their students to participate in CBDE. ${ }^{31} \mathrm{UNC}$ was fortunate to have benefitted from a long history of faculty familiarity with extramural rotations. However, like in other participating Pipeline schools and those that wish to introduce CBDE into their curricula for the first time, some faculty concerns must be addressed. A common concern is one of curricular calibration, or fear that educational — specifically clinical—experiences may be subpar in the community setting relative to experiences at the home institution's on-site dental clinic. ${ }^{26}$ At UNC, the Pipeline team invited the main clinic faculty to extramural clinics to gain a firsthand look at the clinical experiences afforded to students there as well as to meet their community faculty counterparts who precept students at the extramural sites. ${ }^{26}$

Research conducted as part of the national Pipeline program evaluation suggests that support of extramural rotations is more enthusiastic from community faculty members involved in CBDE activities of the initiative than from non-CBDE involved faculty members. ${ }^{32}$ Just as UNC brought school-based faculty members to extramural sites, the authors suggest that their findings indicate that building a partnership between school-based and community faculty is important to the continuation of changes 
in CBDE. A primary goal of CBDE experiences is to prepare students to provide culturally sensitive care in the community. Research conducted as part of the Pipeline program evaluation suggests that faculty members and students must feel that students are prepared to provide sensitive community-based care. Further, the longer students spent in extramural rotations, the greater was faculty confidence that students were well prepared to provide such care. ${ }^{33}$

Schools embarking on increased CBDE through rotational time spent away from the main school clinic must evaluate the potential financial repercussions of such a plan. Considerations include any costs associated with hiring more clinical faculty members in order to have affiliates in the community capable of overseeing students' clinical experiences in those sites. ${ }^{31}$ Some schools, such as UNC, do not financially compensate field preceptors. Other areas of potential difficulty include the costs related to the transportation to and housing at distant sites for students. Perhaps the greatest issue is the extended time students spend away from the main school clinic. Specifically, a worry is that fewer hours in the main school clinics will result in less overall productivity and revenue associated with the care that dental school students provide in in-house clinics. ${ }^{31}$ For UNC, these issues were not seen as barriers during the implementation of increased extramural rotation days; indeed, it was felt that students' time away from the school clinics might reduce the cost for paid, school-based, part-time faculty members who supervise in-house. Concerns about lost inhouse clinic revenue are virtually nonexistent, given that students have been away from the clinic for a significant time throughout the many-year duration of the DISC program. ${ }^{26}$ Research from the Pipeline program evaluation suggests that representatives from participating schools believed that their students increased their skill and treatment speed while away at extramural sites. It was felt by some that this would increase their productivity and revenue generation potential when they returned to the in-house clinic, though no respondents provided financial data and productivity measures to corroborate this belief. ${ }^{30}$

Schools where loss of clinic revenue may seem to be an insurmountable hurdle to increasing CBDE for their students may want to consider a radical change in how they operate their in-house clinics. Shifting to a patient-care delivery model as an opportunity to both increase revenue from in-house clinics and allow students to spend more time away from the school clinic has been discussed. ${ }^{34}$ Such an approach argues that an increased focus on productivity and even further inclusion of challenging patients at school clinics could be achieved if the in-house clinic was not seen primarily as a vehicle for dental student education. When in-house dental school clinics are set up primarily for educational experiences, complex and more revenue-producing cases are screened out in favor of more simple cases that can be handled by dental students albeit at a slower rate than their practitioner colleagues. When varied educational experiences happen at extramural sites, the in-house clinic can be freed up to handle more complex cases and use faculty members to deliver that more highly remunerated care. ${ }^{34}$

UNC's experience with CBDE continues as an integral part of the school culture. Changes accelerated in part by the Pipeline program were consonant with a previous commitment to equality and service to the community. Extramural rotations and curricular and recruitment initiatives are being maintained despite increasing financial constraints. The CBDE-associated gains in student confidence, exposure to and ability to provide service to disadvantaged patients, and positive student and faculty feedback continue to provide the impetus to pursue these integral activities. ${ }^{35}$

On a national and international level, CBDE has been shown to improve dental student learning experiences and has enormous potential to influence values on equity, public service, and collaboration. The growth of CBDE signals the readiness of dentistry to engage with communities and meet the needs of the full range of at-need people in order to enhance the health of the public.

\section{The Broader Impact of CBDE on Students}

Recent studies of the broader impact of CBDE on dental students suggest a promising and complex picture in terms of students' attitudes and plans for caring for the underserved. A broad evaluation of the Pipeline program conducted between 2003 and 2008 found that although practice plans of senior dental students for community-based work were overall unchanged by the program, students' perceptions of the adequacy of their preparation for extramural rotations were positively associated with their plans to provide care to at least 25 percent of underserved patients. ${ }^{31}$ In addition, there was a relationship between student characteristics and plans to provide care: under- 
represented minority students were more than three times as likely to provide care to underserved patients than were other students. Barriers to providing care to underserved patients included educational debt and lack of reimbursement for care to uninsured and publicly insured patients.

Research provides further insight into factors influencing attitudes and practice plans of students who have participated in CBDE. ${ }^{36,37}$ Here again, student characteristics, including personal values and experiences, helped shape attitudes towards treating the underserved. Students who expected to serve more underserved patients were significantly more likely to report the importance of contact with a high school counselor in pursuing dentistry, recruitment by a dental school, and participation in a pre- or postbaccalaureate dental program. Students who were aware of the importance of cultural awareness and who expressed feeling adequately prepared to provide care for racially and culturally diverse groups, expected to spend more weeks in extramural rotations, felt that extramural clinical experiences influenced practice location plans, and planned to work in a community clinic or government service were more likely to report plans to provide care to underserved minorities. In addition, these students were significantly more likely to be female, twenty-two years of age or older, and a member of an underrepresented minority group or an Asian or Pacific Islander. They were also more likely to be unmarried, from a lowerincome family, in a loan repayment program, and a child of a father who did not attend college. They had higher service orientation and social consciousness scores and lower entrepreneurial scores.

A comparison of these findings with other findings from a 2009 study of first-year dental students who participated in a classroom oral health promotion and preventive care program for underserved elementary school children provides further insight into the relationship of CBDE and student experience and personal characteristics. ${ }^{38}$ In the second study, about two-thirds of the 144 freshman dental students were male, younger than twenty-five, and from the suburbs. One-fifth had financial support from parents, and about four-fifths had loans to help pay for dental school. These students were surveyed three times - before, during, and after participation in these programs. Attitudes about societal expectations, health professionals' responsibility, access to care, and students' sense of personal efficacy to positively influence the need for expanded oral health care services for segments of the population were measured. By the end of their first year, students' attitudes still reflected a high degree of social responsibility and care for the underserved. However, as a group they felt less certain that every person has a right to access dental care regardless of ability to pay; that health professionals have the responsibility to fulfill those needs; and that, as dental students, they were capable of providing care for the underserved that would make a difference in the lives of these individuals. Although they continued to believe that society is responsible for providing dental care for the underserved, the first-year students seemed less clear about how these needs should be addressed. The researchers suggest that this uncertainty was possibly a sign of intellectual growth, reflecting their new and better understanding of the complexity of the problem of access to care for the underserved. It also is quite possible that this more sophisticated, if sometimes painful, understanding of the intricate web of the social and economic determinants of oral health would have better equipped them for subsequent experiences in CBDE. In the long term, both kinds of experiences may well generate teachers and community leaders prepared to create the policy, financial, and structural interventions necessary to motivate dental providers to care for the underserved.

In order to assess the impact of externships on dental and dental hygiene students at UNC, an unpublished evaluation was performed before and after experiences over a two-year period. This evaluation study was sponsored by the Kate B. Reynolds Charitable Trust. Before their CBDE experiences, 45 percent of students reported having a clear idea of where they would practice in their future career: 46 percent expected to work in a rural setting; 71 percent expected to treat many underserved patients (e.g., aged, disabled, low socioeconomic status, minority, and institutionalized patients); and 31 percent expected to become a dental specialist.

The evaluation measured perceptions of how CBDE affected students. Thirty-eight percent said that CBDE changed their idea of where they would practice dentistry; 55 percent said their CBDE experience made it more likely that they would stay in North Carolina; 24 percent said that it increased their interest in working in a rural setting; 56 percent said that $\mathrm{CBDE}$ experiences increased their interest in treating underserved patients; 8 percent said CBDE experiences affected their interest in becoming a dental specialist; and 53 percent said that CBDE affected their dental career choice. 
Evaluation has illuminated the ways in which students may benefit from and maximize the impact of their community exposures. Formal evaluation tools should permit students to provide both closedended and open-ended feedback to the program.

\section{Impact of CBDE on Communities}

There is little research on the local impact of CBDE on the communities served by dental students, especially in comparison with the growing literature focused on the impact of CBDE on the educational and personal experiences of dental student. Research could examine issues such as the increase in the number of dental patients served, revenues generated by the student dentists in their community clinic sites, improved oral health education for local populations of all ages, and a gradually broadening community awareness of disease prevention and oral health promotion.

The indirect effects of CBDE on local populations are also worth considering and are likely to be complex. Recent research identified several benefits to extramural dental education as an adjunct to traditional dental school training. ${ }^{39,40} \mathrm{CBDE}$ improved student confidence in tackling clinical situations. It also improved student ability to capture a patient's social history and plan appropriate treatment in the light of that social history. Thus, CBDE can contribute to achieving community public health objectives by helping students move to a more holistic consideration of the patient's needs and increasing their sense of professional competence. The nature of CBDE on the development of empathy in dental students may be more complex and has yet to be evaluated.

A study of first-year U.S. dental students providing classroom oral health promotion and preventive dental care to underserved elementary school children suggests mixed effects regarding social responsibility. ${ }^{38}$ On the one hand, the belief among dental students that society is responsible for providing dental care for the underserved was sustained. However, students seemed less certain about how those needs should be addressed (who should be responsible for fulfilling the obligation, who should receive care, etc.) and their capability to provide this care while in dental school. The researchers point out that these changes in attitude may reflect a greater appreciation for the complexity of the determinants of oral health as a consequence of their communitybased experience. This eye-opening experience may well be an asset to young dentists embarking on practice that incorporates the underserved.

\section{Summary}

Community-based dental education has substantial potential for affecting the values and behaviors for dental students relative to health care access for underserved populations and for attracting a more diverse array of students to dental education. The effective integration of CBDE into a dental curriculum requires specific student preparation in cultural awareness, communication skills, and the social and behavioral sciences. Reflective components, evaluation, and highly organized community-based experiences ensure that student learning is maximized. CBDE also provides dentistry with an opportunity to guide values of the dental faculty and students and orient them towards public service, engagement, ethics, and the health of the public.

\section{Acknowledgments}

The authors would like to express their thanks to Dr. Eugene Sandler (UNC School of Dentistry), who pioneered and led the university's Dentistry in Service to Communities (DISC) Program; Mr. Larry Keith (UNC School of Medicine), who guided the efforts to diversify the UNC Schools of Medicine and Dentistry and who directed the UNC Medical Education Development (MED) Program (Mr. Keith passed away in February 2010); Dr. Janet Southerland (UNC School of Dentistry), who was Co-Principal Investigator of the school's Pipeline program; Dr. Patrena Benton (formerly of UNC, currently Assistant Vice President for Academic Affairs at Langston University in Langston, OK), who was Director of the University of North Carolina Health Careers Access Program; Dr. Janet Guthmiller (UNC School of Dentistry), Associate Dean for Academic Affairs; Dr. Edmond R. Hewlett (UCLA School of Dentistry), who was our external evaluator in the Pipeline program and provided great insight and wisdom about UNC's programs; and Dr. David Brunson, currently Associate Director of the Center for Equity and Diversity, American Dental Education Association, and former Chair of the Admissions Committee at UNC School of Dentistry, who helped facilitate the school's Pipeline program. 


\section{REFERENCES}

1. DeCastro JE, Bolger D, Feldman CA. Clinical competence of graduates of community-based and traditional curricula. J Dent Educ 2005;69(12):1324-31.

2. Littlewood S, Ypinazar V, Margolis SA, Scherpbier A, Spencer J, Dornan T. Early practical experience and the social responsiveness of clinical education: systematic review. BMJ 2005;331(7513):387-91.

3. Dornan T, Littlewood S, Margolis SA, Scherpbier A, Spencer J, Y pinazar V. How can experience in clinical and community settings contribute to early medical education? A BEME systematic review. Med Teacher 2006;28(1):3-18.

4. Woronok JI, Pichbeck YJ, Walter MH. University of Alberta dental students' outreach clinical experience: an evaluation of the program. J Can Dent Assoc 2004;70(4):233-6.

5. Ang M. Advanced communication skills: conflict management and persuasion. Acad Med 2002;77(11):1166.

6. O'Toole TP, Kathuria N, Mishra M, Schukart D. Teaching professionalism within a community context: perspectives from a national demonstration project. Acad Med 2005;80(4):339-43.

7. Wear D, Aultman JM. The limits of narrative: medical student resistance to confronting inequality and oppression in literature and beyond. Med Educ 2005;39(10):1056-65.

8. Holmes DC, Boston DW, Budenz AW, Licari FW. Predoctoral clinical curriculum models at U.S. and Canadian dental schools. J Dent Educ 2003;67(12):1302-11.

9. Kassebaum DK, Hendricson WD, Taft T, Haden NK. The dental curriculum at North American dental institutions in 2002-03: a survey of current structure, recent innovations, and planned changes. J Dent Educ 2004;68(9):914-31.

10. Jacobson J, Tedesco L, Bagramian R, Burgett F, Kotowicz W. Advancing community-based education: curriculum issues. J Dent Educ 1999;63(12):896-901.

11. Strauss R, Mofidi M, Sandler ES, Williamson R 3rd, McMurtry BA, Carl LS, Neal EM. Reflective learning in community-based dental education. J Dent Educ 2003;67(11):1234-42.

12. Mofidi M, Strauss R, Pitner LL, Sandler ES. Dental students' reflections on their community-based experiences: the use of critical incidents. J Dent Educ 2003;67(5):51523.

13. Schwartz AB, Peterson EM, Edelstein BL. Under oath: content analysis of oaths administered in ADA-accredited dental schools in the United States, Canada, and Puerto Rico. J Dent Educ 2009;73(6):746-52.

14. Formicola AJ, McIntosh J, Marshall S, Albert D, MitchellLewis D, Zabos GP, Garfield R. Population-based primary care and dental education: a new role for dental schools. J Dent Educ 1999;63(4):331-8.

15. Whipp JL, Ferguson DJ, Wells LM, Iacopino AM. Rethinking knowledge and pedagogy in dental education. J Dent Educ 2000;64(12):860-6.

16. Donate-Bartfield E, Lausten L. Why practice culturally sensitive care? Integrating ethics and behavioral science. J Dent Educ 2002;66(9):1006-11.
17. Yoshida T, Milgrom P, Coldwell S. How do U.S. and Canadian dental schools teach interpersonal communication skills? J Dent Educ 2002;66(11):1281-8.

18. Balis SA, Rule JT. Humanities in dental education: a focus on understanding the child. J Dent Educ 1999;63(9):709_ 15 .

19. Hottel TL, Hardigan PC. Improvement in the interpersonal communication skills of dental students. J Dent Educ 2005;69(2):281-4

20. American Association of Dental Schools [now the American Dental Education Association]. Proceedings of the 2000 House of Delegates. J Dent Educ 2000;64(7):120319.

21. Mouradian WE, Berg JH, Somerman MJ. Addressing disparities through dental-medical collaborations, Part I. The role of cultural competence in health disparities. J Dent Educ 2003;67(8):860-8.

22. Formicola AJ, Stavisky J, Lewy R. Cultural competence: dentistry and medicine learning from one another. J Dent Educ 2003;67(8):869-75.

23. Betancourt JR. Cross-cultural medical education: conceptual approaches and frameworks for evaluation. Acad Med 2003;8(6):560-9.

24. Mutha SM, Allen A, Welch MD. Towards culturally competent care: a toolbox for teaching culturally competent care. San Francisco: Center for the Health Professions, University of California, San Francisco, 2002.

25. Welch M. Teaching diversity and cross-cultural competence in health care: a trainer's guide. 3rd ed. San Francisco: Perspective of Differences Diversity Training and Consultation Services for Health Professionals, 2003.

26. Hewlett ER, Andersen RM, Atchison KA. The pipeline program at the University of North Carolina at Chapel Hill School of Dentistry. J Dent Educ 2009;73(2 Suppl):S135-45.

27. Leviton LC. Foreword to Evaluating the dental pipeline program: recruiting minorities and promoting community-based dental education. J Dent Educ 2009;73(2 Suppl):S5-7.

28. Hood JG. Service-learning in dental education: meeting needs and challenges. J Dent Educ 2009;73(4):454-63.

29. Andersen RM, Friedman JA, Carreon DC, Bai J, Nakazono TT, Afifi A, Gutierrez JJ. Recruitment and retention of underrepresented minority and low-income dental students: effects of the pipeline program. J Dent Educ 2009;73(2 Suppl):S238-58.

30. Thind A, Atchison KA, Nakazono TT, Gutierrez JJ, Carreon DC, Bai J. Sustainability of dental school recruitment, curriculum, and community-based pipeline initiatives. J Dent Educ 2009;73(2 Suppl):S297-307.

31. Atchison KA, Thind A, Nakazono TT, Wong D, Gutierrez JJ, Carreon DC, Andersen RM. Community-based clinical dental education: effects of the pipeline program. J Dent Educ 2009;73(2 Suppl):S269-82.

32. Thind A, Atchison KA, Andersen RM, Nakazono TT, Gutierrez JJ. Reforming dental education: faculty members' perceptions on the continuation of pipeline program changes. J Dent Educ 2008;72(12):1472-80. 
33. Hewlett ER, Davidson PL, Nakazono TT, Carreon DC, Gutierrez JJ, Afifi A. Revisions to dental school curricula: effects of the pipeline program. J Dent Educ 2009;73(2 Suppl):S259-68.

34. Formicola AJ. Dental school clinics as patient care delivery centers: a paradigm shift in dental education. J Dent Educ 2008;72(2 Suppl):S18-20.

35. Strauss RP. Comments from the University of North Carolina at Chapel Hill School of Dentistry. J Dent Educ 2009;73(2 Suppl):S145-7.

36. Davidson PL, Carreon DC, Baumeister SE, Nakazono TT, Gutierrez JJ, Afifi A, Andersen RM. Influence of contextual environment and community-based dental education on practice plans of graduating seniors. J Dent Educ 2007;71(3):403-18.
37. Davidson PL, Nakazono TT, Carreon DC, Bai J, Afifi A. Practice plans of dental school graduating seniors: effects of the pipeline program. J Dent Educ 2009;73(2 Suppl):S283-96.

38. Holtzman JS, Seirawan H. Impact of communitybased oral health experiences on dental students' attitudes towards caring for the underserved. J Dent Educ 2009; 73(3):303-10.

39. Smith M, Lennon MA, Brook AH, Robinson PG. A randomized controlled trial of outreach placement's effect on dental students' clinical confidence. J Dent Educ 2006;70(5):566-70.

40. Smith M, Lennon MA, Brook AH, Blinkhorn FA, Blinkhorn AS, Robinson PG. A randomized controlled trial of the effect of outreach placement on treatment planning by dental students. Br Dent J 2006;Suppl:27-31. 